\title{
A COMPLETE DESCRIPTION OF DYNAMICS GENERATED BY BIRTH-AND-DEATH PROBLEM: A SEMIGROUP APPROACH
}

\author{
JACEK BANASIAK \\ School of Mathematical and Statistical Sciences \\ University of Natal, Durban 4041, South Africa \\ E-mail: banasiak@nu.ac.za
}

\begin{abstract}
We shall present necessary and sufficient conditions for both conservativity and uniqueness of solutions to birth-and-death system of equations using methods of semigroup theory. The derived conditions correspond to the uniqueness criteria for forward and backward birth-and-death systems due to Reuter, $[10,11,1]$, that were derived in a different context by Markov processes' techniques.
\end{abstract}

1. Introduction. In order to provide a meaningful introduction to this paper let us first provide a brief description of the model and introduce the necessary notation.

1.1. The model. We shall consider the classical Markov birth-and-death process that describes the evolution of the population whose size $k$ at any time $t$ may increase to $k+1$ or decrease to $k-1$ owing to a "birth" or a "death" of an individual; the probability that a birth or death occur in time interval $\Delta t$ being $\lambda_{k} \Delta t+o(\Delta t)$ and $\mu_{k} \Delta t+o(\Delta t)$, respectively. If we denote by $u_{k}(t)$ the probability that the population is of size $k$ at time $t$, then the corresponding (forward) Kolmogorov system takes the form:

$$
\begin{aligned}
u_{0}^{\prime} & =-\lambda_{0} u_{0}+\mu_{1} u_{1}, \\
& \vdots \\
u_{n}^{\prime}= & -\left(\lambda_{n}+\mu_{n}\right) u_{n}+\mu_{n+1} u_{n+1}+\lambda_{n-1} u_{n-1},
\end{aligned}
$$

We shall use the convention that boldface letters denote sequences, e.g. $\mathbf{u}=\left(u_{0}, u_{1}, \ldots\right.$, $\left.u_{n}, \ldots\right)$. Also, we put $\lambda_{-1}=\mu_{0}=0$ and, to avoid technicalities (see e.g. [1, p. 100]) we assume that $\lambda_{n}, \mu_{n}>0$ for all other indices. $\mathbb{N}_{0}$ denotes the set $\{0,1,2, \ldots\}$.

2000 Mathematics Subject Classification: Primary 47D06; Secondary 60J80.

Research supported by National Research Foundation of South Africa.

The paper is in final form and no version of it will be published elsewhere. 
System (1) is considered in the Banach space $X=l^{1}$ — this choice is dictated by the fact that the probabilistic interpretation of $\mathbf{u}$ yields $u_{k} \geq 0$ and

$$
\|\mathbf{u}\|_{X}=\sum_{k=0}^{\infty} u_{k}=1
$$

so that the $X$-norm of $\mathbf{u}$ should be preserved in the evolution.

For any subspace $Z$ of $X, Z_{+}$denote the cone of nonnegative elements of $Z$.

It is convenient to write the right-hand side of (1) as the sum of two operators. Firstly we introduce formal mappings of sequences. Remembering the convention $\lambda_{-1}=\mu_{0}=0$, we let $\mathbf{w}=\mathcal{A} \mathbf{u}=-\left\{\left(\lambda_{n}+\mu_{n}\right) u_{n}\right\}_{n \in \mathbb{N}_{0}}$. By $\mathcal{B}$ we denote the mapping $\mathbf{v}=\mathcal{B} \mathbf{u}$, where $\mathbf{v}=\left\{\mu_{n+1} u_{n+1}+\lambda_{n-1} u_{n-1}\right\}_{n \in \mathbb{N}_{0}}$. The formal mappings $\mathcal{A}$ and $\mathcal{B}$ can define various operators in $X$. As a basic choice we define the operator $A$ in $X$ as the restriction of $\mathcal{A}$ to the domain

$$
D_{A}=\{\mathbf{u} \in X ; \mathcal{A} \mathbf{u} \in X\}
$$

In particular, if $\mathbf{u} \in D(A)_{+}$, then a simple calculation shows that $\mathbf{v}=\mathcal{B} \mathbf{u} \in X_{+}$with

$$
\sum_{n=0}^{\infty}\left(v_{n}+w_{n}\right)=0
$$

This allows us to define a positive operator $B$ as the restriction of $\mathcal{B}$ to $D_{A}$. It follows then that for $\mathbf{u} \in D_{A}$ we have

$$
\|B \mathbf{u}\| \leq\|A \mathbf{u}\|
$$

1.2. The description of the results. Mathematical equations of the applied sciences are built by combining various conservation and constitutive laws. They are also formulated and understood pointwise, that is, all the operations, like differentiation, summation or integration, are meant in the classical "calculus" sense, and the equation itself is supposed to be satisfied for all reasonable values of the independent variables. Thus the birth-and-death system (1) is basically understood as

$$
\mathbf{u}^{\prime}=\mathcal{A} \mathbf{u}+\mathcal{B} \mathbf{u}
$$

that is, the equations of the system, taken row by row, should be satisfied for all $\mathbf{u}$ for which the expression above makes sense. Only the probabilistic interpretation of the solution suggests that one should have $u_{n}(t) \geq 0$ for all $n \in \mathbb{N}_{0}$ and $t \geq 0$, and

$$
\sum_{n=0}^{\infty} u_{n}(t)=\sum_{n=0}^{\infty} u_{n}(0)=1, \quad t>0
$$

However, when we prove the solvability of (5), its meaning often changes to make it manageable by particular techniques. Thus, if we prove the existence of a semigroup (dynamical system) "solving" (5), then what we really obtain is a solution to a particular reformulation of the original problem, where on the right-hand side stands the generator $T$ of this semigroup which (at least in the linear case) is uniquely defined. This generator may be quite different from $\mathcal{A}+\mathcal{B}$ and only a detailed characterization of its domain can reveal whether the constructed semigroup gives the full picture of the dynamics described by (5). For the birth-and-death system it can be proved that the generator 
$T$ is between the minimal operator $T_{\min }=A+B$ (defined on $\left.D(A)\right)$ and the maximal operator $T_{\max }=\mathcal{A}+\mathcal{B}$ defined on

$$
D_{\max }=\{\mathbf{u} \in X ; \mathcal{A} \mathbf{u}+\mathcal{B} \mathbf{u} \in X\}
$$

that is,

$$
T_{\min } \subseteq T \subseteq T_{\max }
$$

The position of $T$ on this scale determines the well-posedness of the problem (5). The following situations are possible and are of interest here:

1. $T_{\min }=T=T_{\max }$,

2. $T_{\min } \varsubsetneqq T=\overline{T_{\min }}=T_{\max }$,

3. $T_{\min }=T \varsubsetneqq T_{\max }$,

4. $T_{\min } \varsubsetneqq T=\overline{T_{\min }} \varsubsetneqq T_{\max }$,

5. $\overline{T_{\min }} \varsubsetneqq T \varsubsetneqq T_{\max }$.

It turns out that each case has its own specific interpretation in the model.

In all cases where $T \varsubsetneqq T_{\max }$ we don't have uniqueness, that is, there are strongly differentiable $X$-valued solutions to (5) emanating from zero and therefore they are not described by the constructed dynamical system: "there is more to life than meets the semigroup" $[9,4]$. To achieve uniqueness here one has to impose additional constraints on the solution.

If $\overline{T_{\min }} \varsubsetneqq T$, then despite the fact that the model is formally conservative, see (3), the solutions are not - the described quantity leaks out from the system and the mechanism of this leakage is not present in the model. In the Markov processes' terminology such case is referred to as dishonesty of the transition function, $[11,1]$.

Finally, as $\lambda_{n}, \mu_{n}$ are the rates of change of the states in the population, for any solution $\mathbf{u}(t)$ the quantity

$$
\Delta t \sum_{n=0}^{\infty}\left(\lambda_{n}+\mu_{n}\right) u_{n}(t)
$$

describes the total number of state changes in the interval $\Delta t$. Thus the condition $\mathbf{u}(t) \in$ $D(A)$ for any $t$, equivalent to (6) being finite, reflects the realistic property of finite total number of "switches" at any time. Thus, if $T \neq T_{\min }$, then there may occur infinite number of state changes in a finite time interval.

Therefore, strictly speaking only the problems with $T=T_{\min }=T_{\max }$ can be physically realistic. However, in many applications the first equality is deemed to be not so important and the case $T=\overline{T_{\min }}=T_{\max }$ is considered to be "optimal". We shall follow this line in the paper and thus we shall not address the question under what conditions $u(t) \in D(A)$ for $t \geq 0$ but we shall prove necessary and sufficient conditions for $T=\overline{T_{\min }}$ and $T=T_{\max }$. We shall also compare our results with those existing in the literature.

Acknowledgements. The author is greatly indebted to Dr A. Bobrowski for stimulating discussions during the conference that made the author more aware of the probabilistic context of the problem and, in particular, for mentioning the monograph [1]. 
2. Reuter-Lederman method. In this section we shall reformulate the classical result of Reuter and Lederman (e.g. [10]) for solving Kolmogorov equations in terms of the semigroup theory. The idea of this method is to approximate the solution of (1) by a sequence of solutions of cut-off problems of a similar form.

For the sequence $\mathbf{u} \in X$ we introduce the projection operators

$$
P_{n} \mathbf{u}= \begin{cases}u_{i} & \text { if } 0 \leq i \leq n \\ 0 & \text { if } i>n\end{cases}
$$

define $A_{n}=A P_{n}=P_{n} A=P_{n} A P_{n}$ and $B_{n}=P_{n} B P_{n}$, and consider the system of ordinary differential equations in $\mathbb{R}^{n}$

$$
\mathbf{u}_{n}=A_{n} \mathbf{u}_{n}+B_{n} \mathbf{u}_{n} .
$$

The right-hand side generates a uniformly continuous positive semigroup of contractions on $\mathbb{R}^{n}$, denoted by $\left(G_{n}(t)\right)_{t \geq 0}$. The family $\left(G_{n}(t)\right)_{t \geq 0}$ can be extended to the uniformly continuous family of operators defined on the whole of $X$ by $\bar{G}_{n}(t)=P_{n} G_{n}(t) P_{n}$. Note that $\left(\bar{G}_{n}(t)\right)_{t \geq 0}$ is no longer a semigroup.

TheOrem 1. (a) There is a positive $C_{0}$-semigroup of contractions $(G(t))_{t \geq 0}$ such that for $\stackrel{\circ}{\mathbf{u}} \in X$ and $t \geq 0$

$$
G(t) \stackrel{\circ}{\mathbf{u}}=\lim _{n \rightarrow \infty} \bar{G}_{n}(t) \stackrel{\circ}{\mathbf{u}},
$$

and the generator $\mathfrak{T}$ of $(G(t))_{t \geq 0}$ is an extension of $T_{\min }$.

(b) If $t \rightarrow \mathbf{v}(t)=\left(v_{1}(t), v_{2}(t) \ldots\right)$ is a sequence of functions such that for any $k, t \rightarrow v_{k}(t)$ is integrable on any bounded subset of $\mathbb{R}_{+}$and satisfies for almost all $t$ and any $k$ :

$$
v_{k}(t)=\stackrel{\circ}{u}_{k}+\int_{0}^{t}\left(-\left(\lambda_{k}+\mu_{k}\right) v_{k}(s)+\lambda_{k-1} v_{k-1}(s)+\mu_{k+1} v_{k+1}(s)\right) d s,
$$

then for all $t \geq 0$ and all $k$

$$
v_{k}(t) \geq(G(t) \stackrel{\circ}{\mathbf{u}})_{k}
$$

(c) For any $\stackrel{\circ}{\mathbf{u}} \in X, \mathbf{u}(t)=G(t) \stackrel{\circ}{\mathbf{u}}$ satisfies the equation (10) for any $t \geq 0$ and every $k$.

Investigation of the conservativity of solutions was reduced, [10, Theorems 6 and 7], also $[6,12]$, to the analysis of summability of the expression $w_{n, n_{0}}=\bar{w}_{n, n_{0}}+\tilde{w}_{n, n_{0}}$, where

$$
\begin{aligned}
\bar{w}_{n, n_{0}} & =\frac{1}{\lambda_{n}}+\frac{\mu_{n}}{\lambda_{n} \lambda_{n-1}}+\ldots+\frac{\mu_{n} \cdots \mu_{n_{0}+1}}{\lambda_{n} \cdots \lambda_{n_{0}}}, \\
\tilde{w}_{n, n_{0}} & =\frac{\mu_{n} \cdots \mu_{n_{0}}}{\lambda_{n} \cdots \lambda_{n_{0}}} .
\end{aligned}
$$

THEOREM 2. Let us denote by $\mathbf{u}_{n_{0}}(t)$ the solution corresponding to the initial condition $\stackrel{\circ}{\mathbf{u}}_{n_{0}}$ defined by $\stackrel{\circ}{u}_{n, n_{0}}=\delta_{n, n_{0}}$ (Kronecker's delta).

(a) If

$$
\sum_{n=n_{0}}^{\infty} w_{n, n_{0}}=\infty
$$

then $\sum_{n=0}^{\infty} u_{n, n_{0}}(t)=1$ for all $t \geq 0$. 
(b) If

$$
\sum_{n=n_{0}}^{\infty} w_{n, n_{0}}<\infty \quad \text { and } \quad \mu_{n+1} w_{n, n_{0}}=O(1),
$$

then $\sum_{n=0}^{\infty} u_{n, n_{0}}(t)<1$ for some $t>0$.

3. Kato-Voigt perturbation method. Let us recall the assumptions of Voigt's result in an abstract situation. Let $X$ denote the Banach space $L_{1}(\Omega, \mu)$, where $(\Omega, \mu)$ is a measure space, endowed with the standard norm $\|\cdot\|$. In particular, as in this paper, we may have $\Omega=\mathbb{N}_{0}$ with $\mu$ being the counting measure. Let $(G(t))_{t \geq 0}$ be a strongly continuous semigroup on $X$. We say that $(G(t))_{t \geq 0}$ is a substochastic semigroup if for each $t \geq 0, G(t) \geq 0$ and $\|G(t)\| \leq 1$. It is called a stochastic semigroup if additionally $\|G(t) f\|=\|f\|$ for $X_{+}$. We shall consider two linear operators in $X: A$ and $B$, that have the properties:

1. $(A, D(A))$ generates substochastic semigroup $\left(G_{A}(t)\right)_{t \geq 0}$,

2. $D(B) \supset D(A)$ and $B f \geq 0$ for any $f \in D(B)_{+}$,

3. for any $f \in D(A)_{+}$

$$
\int_{\Omega}(A f+B f) d \mu \leq 0
$$

In this context we have the following basic theorem $[13,5]$

THEOREM 3. Under the above assumptions there exists a smallest substochastic semigroup $\left(G_{T}(t)\right)_{t \geq 0}$ generated by an extension $T$ of $(A+B, D(A))$. This semigroup can be obtained as the strong limit in $X$ of semigroups $\left(G_{r}(t)\right)_{t \geq 0}, 0<r<1$, generated by $(A+r B, D(A))$. The convergence is monotonic as $r \nearrow 1^{-}$. Moreover, $\left(G_{T}(t)\right)_{t \geq 0}=$ $(G(t))_{t \geq 0}$, where $(G(t))_{t \geq 0}$ is the semigroup constructed by the Reuter-Lederman method.

We shall find whether the constructed semigroup is conservative by means of the technique introduced by Arlotti in [2] and developed in a few subsequent papers [3, 5]. The main result of [2] specified to the present situation reads:

TheOREM 4. If for any $\mathbf{u} \in X_{+}$such that $\mathcal{A} \mathbf{u}+\mathcal{B} \mathbf{u} \in X$ we have

$$
\sum_{n=0}^{\infty}\left(-\left(\lambda_{n}+\mu_{n}\right) u_{n}+\lambda_{n-1} u_{n-1}+\mu_{n+1} u_{n+1}\right) \geq 0
$$

then $T=\overline{A+B}$.

Then we have the following result:

TheOREM 5. $T=\overline{A+B}$ if and only if

$$
\sum_{n=0}^{\infty} \frac{1}{\lambda_{n}}\left(\sum_{i=0}^{\infty} \prod_{j=1}^{i} \frac{\mu_{n+j}}{\lambda_{n+j}}\right)=+\infty
$$

(where we put $\prod_{j=1}^{0}=1$ ). 
Proof. Assume, on the contrary, that for some $0 \leq \mathbf{u} \in D_{\max }$ the sum in (17) is negative, then there is $b>0$ such that the convergent sequence

$$
\begin{aligned}
b_{n} & =-\lambda_{n} u_{n}+\mu_{n+1} u_{n+1} \\
& =\lim _{n \rightarrow \infty} \sum_{k=0}^{n}\left(-\left(\lambda_{n}+\mu_{n}\right) u_{n}+\lambda_{n-1} u_{n-1}+\mu_{n+1} u_{n+1}\right),
\end{aligned}
$$

satisfies $b_{n} \leq-b$ for all $n \geq n_{0}$ with large enough $n_{0}$. We can easily modify $\mathbf{u}$ so that all $b_{n}$-s are less or equal than $-b$. This can be done e.g. by putting $u_{k}=\lambda_{k}^{-1}\left(b+\mu_{k+1} u_{k+1}\right)$ for $0 \leq k \leq n_{0}-1$ and leaving $u_{k}$ with $k \geq n_{0}$ unchanged. Clearly such a redefined $\mathbf{u}$ satisfies $0 \leq \mathbf{u} \in D_{\max }$.

Starting from (19) we get for $n \geq 0$

$$
u_{n} \geq \frac{b}{\lambda_{n}}+\frac{\mu_{n+1}}{\lambda_{n}} u_{n+1},
$$

and, by induction, for arbitrary $k$

$$
u_{n} \geq \frac{b}{\lambda_{n}}\left(\sum_{i=0}^{k} \prod_{j=1}^{i} \frac{\mu_{n+j}}{\lambda_{n+j}}\right)+\frac{\mu_{n+1} \ldots \mu_{n+k+1}}{\lambda_{n} \ldots \lambda_{n+k}} u_{n+k+1} .
$$

Since $k$ is arbitrary, we obtain in particular

$$
u_{n} \geq \frac{b}{\lambda_{n}}\left(\sum_{i=0}^{\infty} \prod_{j=1}^{i} \frac{\mu_{n+j}}{\lambda_{n+j}}\right)
$$

and, if the assumption (18) is satisfied, we obtain $\sum_{n=0}^{\infty} u_{n}=+\infty$, which contradicts the assumption of summability of $\left\{u_{n}\right\}_{n \in \mathbb{N}_{0}}$.

To prove the necessity let us assume that the series in (18) is convergent and observe that

$$
\sum_{n=0}^{\infty} \frac{1}{\lambda_{n}}\left(\sum_{i=0}^{\infty} \prod_{j=1}^{i} \frac{\mu_{n+j}}{\lambda_{n+j}}\right)=\frac{1}{\lambda_{0}} \sum_{l=0}^{\infty} \prod_{i=0}^{l-1} \frac{\lambda_{i}}{\mu_{i+1}}\left(\sum_{r=l}^{\infty} \prod_{j=1}^{r} \frac{\mu_{j}}{\lambda_{j}}\right)
$$

Let us construct $\mathbf{u}$ so that for $n \geq 0$ and some $b>0$,

$$
-b=-\lambda_{n} u_{n}+\mu_{n+1} u_{n+1} .
$$

Using e.g [7, Eq. (1.2.4)], we get for $n \geq 1$

$$
u_{n}=\prod_{i=0}^{n-1} \frac{\lambda_{i}}{\mu_{i+1}}\left(u_{0}-\frac{b}{\lambda_{0}} \sum_{l=0}^{n-1} \prod_{i=1}^{l} \frac{\mu_{i}}{\lambda_{i}}\right)
$$

From the assumption we have in particular that the series $\sum_{l=0}^{\infty} \prod_{i=1}^{l} \mu_{i} / \lambda_{i}$ is convergent as the internal series of a convergent double series of positive elements. Moreover, this series converges monotonically so if we define $u_{0}=\left(b / \lambda_{0}\right) \sum_{l=0}^{\infty} \prod_{i=1}^{l} \mu_{i} / \lambda_{i}$, then $u_{n}$ s so defined are nonnegative and are given by the formula

$$
u_{n}=\frac{b}{\lambda_{0}} \prod_{i=0}^{n-1} \frac{\lambda_{i}}{\mu_{i+1}}\left(\sum_{l=n}^{\infty} \prod_{i=1}^{l} \frac{\mu_{i}}{\lambda_{i}}\right) .
$$

By (20) and (18) we obtain that $\sum_{n=0}^{\infty} u_{n}<\infty$ and by construction, $\mathcal{A} \mathbf{u}+\mathcal{B} \mathbf{u} \in l^{1}$, so that $\mathbf{u}$ belongs to the domain of the maximal operator. We must find an element 
with the same properties but in $D(T)$. First, we find $\mathbf{f}=\mathbf{u}-(\mathcal{A} \mathbf{u}+\mathcal{B} \mathbf{u})$. We obtain $f_{0}=u_{0}+\lambda_{0} u_{0}-\mu_{1} u_{1}=u_{0}+b$ and for $n>0$,

$$
f_{n}=u_{n}+\lambda_{n} u_{n}+\mu_{n} u_{n}-\lambda_{n-1} u_{n-1}-\mu_{n+1} u_{n+1}=u_{n},
$$

so that $0 \leq \mathbf{f} \in l^{1}$. For any sequence $\mathbf{u}$, the mapping $\mathcal{L} \mathbf{u}=\left(\left(1+\lambda_{n}+\mu_{n}\right)^{-1} u_{n}\right)_{n \geq 0}$ is well-defined and for $\mathbf{u} \in l^{1}$ we have $\mathcal{L} \mathbf{u}=R(1, A) \mathbf{u}$, where $A$ was defined in (2). Since also the mapping $\mathcal{B}$ is defined for any sequence, we can write the system of equations

$$
\mathbf{u}-\mathcal{A} \mathbf{u}-\mathcal{B} \mathbf{u}=\mathbf{f}
$$

as

$$
\mathbf{u}-\mathcal{L} \mathcal{B} \mathbf{u}=\mathcal{L} \mathbf{f}
$$

and consider all iterates for $n \geq 1$

$$
(\mathcal{L B})^{n} \mathbf{u}-(\mathcal{L B})^{n+1} \mathbf{u}=(\mathcal{L B})^{n} \mathcal{L} \mathbf{f}=R(1, A)(B R(1, A))^{n} \mathbf{f},
$$

where in the last term we used the fact that on $l^{1}$ the mapping $\mathcal{L}$ coincides with $R(1, A)$ and that $D(B) \supset D(A)$. Summing up the iterates we obtain

$$
\mathbf{u}-(\mathcal{L B})^{n+1} \mathbf{u}=\sum_{i=0}^{n} R(1, A)(B R(1, A))^{n} \mathbf{f} .
$$

From the general theory of substochastic semigroups (e.g. [3]) we obtain that the righthand side converges in the norm to a (positive) element $\mathbf{u}_{g}$ of the domain of the generator and, since we have found that $\mathbf{u}$ is nonnegative, the sequence of iterates also converges in the norm to a nonnegative element $\mathbf{h}$ which belongs to the kernel of $I-(\mathcal{A}+\mathcal{B})$. Thus

$$
0 \leq \mathbf{u}_{g}=\mathbf{u}-\mathbf{h}
$$

and

$$
-b=\sum_{i=0}^{\infty}((\mathcal{A}+\mathcal{B}) \mathbf{u})_{i}=\sum_{i=0}^{\infty}\left(T \mathbf{u}_{g}\right)_{i}+\sum_{i=0}^{\infty}((\mathcal{A}+\mathcal{B}) \mathbf{h})_{i}=\sum_{i=0}^{\infty}\left(T \mathbf{u}_{g}\right)_{i}+\sum_{i=0}^{\infty} h_{i} .
$$

Since $\mathbf{h}$ is nonnegative, we obtain $\sum_{i=0}^{\infty}\left(T \mathbf{u}_{g}\right)_{i}<0$. Next, let us consider

$$
\|\mathbf{f}\|=\left\|(I-T) \mathbf{u}_{g}\right\|=\sum_{i=0}^{\infty} u_{g, i}-\sum_{i=0}^{\infty}\left(T \mathbf{u}_{g}\right)_{i} \geq\left\|\mathbf{u}_{\mathbf{g}}\right\|+b
$$

which can be expressed as $\left\|(I-T)^{-1} \mathbf{f}\right\|<\|\mathbf{f}\|$, which by [3, 8] contradicts the conservativity of the semigroup and makes $T=\overline{A+B}$ impossible.

Next we shall relate this result to the conditions of [10, Theorem 6], also Theorem 2. Proposition 1. Condition (18) is equivalent to (14) for some (any) $n_{0} \geq 0$. Thus the sufficient condition for conservativity of the solution from [10] is also necessary.

Proof. Let us denote the inner sum appearing in (18) by $W_{n}=\sum_{r=0}^{\infty} a_{n, r}$ where

$$
a_{n, 0}=\frac{1}{\lambda_{n}}, \quad a_{n, r}=\frac{\mu_{n+1} \cdot \ldots \cdot \mu_{n+r}}{\lambda_{n} \lambda_{n+1} \cdot \ldots \cdot \lambda_{n+r}} .
$$

Next, $\bar{w}_{n, n_{0}}$ of (12) can be written as $\bar{w}_{n, n_{0}}=\sum_{s=0}^{n-n_{0}} b_{n, s}$ with

$$
b_{n, 0}=\frac{1}{\lambda_{n}}, \quad \frac{\mu_{n} \cdot \ldots \cdot \mu_{n-s+1}}{\lambda_{n} \cdot \ldots \cdot \lambda_{n-s}},
$$


thus changing the variable $n$ according to $n=s+l$ ( $l$ is the new variable) we obtain for $s>0$

$$
b_{n, s}=b_{l+s, s}=\frac{\mu_{s+l} \cdot \ldots \cdot \mu_{l+1}}{\lambda_{s+l} \cdot \ldots \cdot \lambda_{l}}=a_{l, s}
$$

and for $s=0, b_{n, 0}=1 / \lambda_{l}=a_{l, 0}$. Therefore, for any $n_{0}$

$$
\sum_{n=n_{0}}^{\infty} \bar{w}_{n, n_{0}}=\sum_{n=n_{0}}^{\infty} \sum_{s=0}^{n-n_{0}} b_{n, s}=\sum_{s=0}^{\infty} \sum_{l=n_{0}}^{\infty} b_{l+s, s}=\sum_{s=0}^{\infty} \sum_{l=n_{0}}^{\infty} a_{l, s}=\sum_{l=n_{0}}^{\infty} W_{l} .
$$

Let us fix $n_{0}$. If (18) is satisfied, then either $\sum_{l=n_{0}}^{\infty} W_{l}=\infty$, or for some $0 \leq k \leq n_{0}-1$ $A_{k}:=\sum_{l=k}^{\infty} \prod_{i=k+1}^{l} \mu_{i} / \lambda_{i}=+\infty$. Let $m>k$. Then

$$
A_{k}:=1+\sum_{l=k}^{m-1} \prod_{i=k+1}^{l} \frac{\mu_{i}}{\lambda_{i}}+A_{m} \prod_{i=k+1}^{m} \frac{\mu_{i}}{\lambda_{i}}
$$

so that $A_{k}$ and $A_{m}$ are simultaneously either convergent or divergent for any pair $k, m$. Using this result together with (22) we see that the Reuter-Ledermann condition (14) is satisfied for any $n_{0}$.

Conversely, if the Reuter-Lederman condition is satisfied even for some $n_{0}$, then either $\sum_{n=n_{0}}^{\infty} \bar{w}_{n, n_{0}}=\sum_{l=n_{0}}^{\infty} W_{l}=+\infty$ or $\sum_{n=n_{0}}^{\infty} \tilde{w}_{n, n_{0}}=A_{n_{0}}=\infty$. This yields (18).

REMARK 1. In [1, Chapter 3, Theorem 2.2] the condition (18) appears in a different context: as the necessary and sufficient condition for the minimal solution to the backward equation to be its unique solution. The link between this statement and the conservativity (honesty) of solutions to the forward equation is not explicitly indicated. However, as the matrices of the coefficient of forward and backward equations are (at least formally) transpose to each other, such a link can be established on the basis of the results relating conservativity of substochastic semigroups and the structure of the point spectrum of the adjoint problem, [8].

4. Maximality of the generator. Let us use the general setting of Section 3. First we state a simple observation that will be the basis of our considerations.

Proposition 2. Let $(G(t))_{t \geq 0}$ be a substochastic semigroup generated by $T$. If for some $0 \leq h \in D\left(T_{\max }\right)$

$$
\int_{\Omega} T_{\max } h d \mu>0
$$

then $T \neq T_{\max }$.

Conversely, assume that

(A1) either

$$
N_{\sigma}:=\bigcup_{\sigma>0} \operatorname{ker}(\sigma I-(\mathcal{A}+\mathcal{B})) \cap X=\emptyset
$$

or, if $N_{\sigma} \neq \emptyset$, then $N_{\sigma}^{+}=N_{\sigma} \cap X_{+} \neq \emptyset$ and

$$
\int_{\Omega} T_{\max } h d \mu=0
$$

for any $h \in D\left(T_{\max }\right)$, then $T_{\max }=T$. 
Proof. Since $T$ generates a substochastic semigroup, it is dissipative, thus if $0 \leq h \in$ $D(T)$, then $\int_{\Omega} T h d \mu \leq 0$. Since $T \subset T_{\max },(23)$ shows that $h \notin D(T)$.

If $T_{\max } \neq T$, then by [4] and (A1), $N_{\sigma}^{+} \neq \emptyset$. Taking $0 \neq h \in N_{\sigma}^{+}$, we get

$$
\int_{\Omega} T_{\max } h d \mu=\sigma \int_{\Omega} h d \mu>0
$$

contradicting (24).

Lemma 1. If $N_{\sigma} \neq \emptyset$, then there is $0 \leq h \in N_{\sigma}$.

Proof. If $h=\left\{h_{n}\right\}_{n \in \mathbb{N}_{0}} \in N_{\sigma}$, then it must be a formal solution to

$$
\begin{aligned}
\sigma h_{0} & =-h_{0} \lambda_{0}+\mu_{1} h_{1}, \\
& \vdots \\
\sigma h_{n} & =-h_{n}\left(\lambda_{n}+\mu_{n}\right)+\mu_{n+1} h_{n+1}+\lambda_{n-1} h_{n-1},
\end{aligned}
$$

with $\sigma>0$. Let us first note that as $\mu_{1} h_{1}=\left(\sigma+\lambda_{0}\right) h_{0}, h_{1} \geq 0$ provided $h_{0} \geq 0$ so that

$$
\left(\sigma+\mu_{1}\right) h_{1}-\lambda_{0} h_{0}=\lambda\left(h_{1}+h_{0}\right) \geq 0
$$

For arbitrary $k>1$ we have from $(26)$

$$
\begin{aligned}
\left(\sigma+\mu_{k}\right) h_{k}-\lambda_{k-1} h_{k-1} & =\left(1+\frac{\sigma}{\mu_{k}}\right)\left(\left(\sigma+\lambda_{k-1}+\mu_{k-1}\right) h_{k-1}-\lambda_{k-2} h_{k-2}\right)-\lambda_{k-1} h_{k-1} \\
(27) \quad & \geq\left(1+\frac{\sigma}{\mu_{k}}\right)\left(\left(\sigma+\mu_{k-1}\right) h_{k-1}-\lambda_{k-2} h_{k-2}\right)
\end{aligned}
$$

so that by induction $\left(\sigma+\mu_{k}\right) h_{k}-\lambda_{k-1} h_{k-1} \geq 0$ for any $k$. Thus $h_{k} \geq \frac{\lambda_{k-1} h_{k-1}}{\sigma+\mu_{k}} \geq 0$. THEOREM 6. $T \neq T_{\max }$ if and only if

$$
\sum_{n=1}^{\infty} \frac{1}{\mu_{n}} \prod_{j=1}^{n-1} \frac{\lambda_{j}}{\mu_{j}}\left(\sum_{i=0}^{n-1} \frac{\mu_{i}}{\lambda_{i}}\right)<+\infty
$$

Proof. Due to Lemma 1 and Proposition $2, T \neq T_{\max }$ if and only if for each $\left\{u_{n}\right\}_{n \in \mathbb{N}_{0}} \in l_{+}^{1}$ such that $\left\{-\left(\lambda_{n}+\mu_{n}\right) u_{n}+\lambda_{n-1} u_{n-1}+\mu_{n+1} u_{n+1}\right\}_{n \in \mathbb{N}_{0}} \in l^{1}$ we have

$$
I=\sum_{n=0}^{\infty}\left(-\left(\lambda_{n}+\mu_{n}\right) u_{n}+\lambda_{n-1} u_{n-1}+\mu_{n+1} u_{n+1}\right)>0 .
$$

As in (19) we have to investigate the behaviour of the sequence $\left\{r_{n}\right\}_{n \in \mathbb{N}_{0}}$ defined as

$$
r_{n}=-\lambda_{n} u_{n}+\mu_{n+1} u_{n+1}, \quad n \geq 0 .
$$

Using again [7, Eq. (1.2.4)] we obtain for $n \geq 1$

$$
u_{n}=\frac{1}{\mu_{n}} \sum_{i=0}^{n-1}\left(r_{i} \prod_{j=1}^{n-1-i} \frac{\lambda_{n-j}}{\mu_{n-j}}\right)+\frac{u_{0} \lambda_{0}}{\mu_{n}} \prod_{j=1}^{n-1} \frac{\lambda_{j}}{\mu_{j}} .
$$


Factoring out $g_{n-1}:=\prod_{j=1}^{n-1} \frac{\lambda_{j}}{\mu_{j}}$ from (30) we can rewrite $u_{n}$ in the more compact form

$$
u_{n}=\frac{g_{n-1}}{\mu_{n}}\left(r_{0}+\lambda_{0} u_{0}+\sum_{i=1}^{n} r_{i} g_{i}^{-1}\right) \text {. }
$$

If $T \neq T_{\max }$, then there is a nonnegative $\left\{u_{n}\right\}_{n \in \mathbb{N}_{0}} \in l^{1}$ for which $I=\lim _{n \rightarrow \infty} r_{n}>0$. Thus the terms of $\left\{r_{n}\right\}_{n \in \mathbb{N}_{0}}$ are strictly positive starting from some $n_{0}^{\prime}$. If $n_{0}^{\prime}>0$, then we can modify $\left\{u_{n}\right\}_{n \in \mathbb{N}_{0}}$ so that $r_{n}>0$ also for $0 \leq n \leq n_{0}^{\prime}-1$. Indeed, first observe that there is $n_{0}^{\prime \prime} \geq n_{0}$ with $u_{n_{0}^{\prime \prime}}>0$ (otherwise all $u_{n}$ are zero starting from $n_{0}$ which gives $r_{n}=0$ for $\left.n \geq n_{0}\right)$. Taking $n_{0}=\max \left\{n_{0}^{\prime}, n_{0}^{\prime \prime}\right\}$, we modify $\left\{u_{n}\right\}_{n \in \mathbb{N}_{0}}$ by putting

$$
\bar{u}_{n_{0}}=u_{n_{0}}, \quad 0<\bar{u}_{n_{0}-k}<\frac{\mu_{n_{0}+1-k}}{\lambda_{n_{0}-k}} \bar{u}_{n_{0}+1-k} \quad \text { for } k=0, \ldots n_{0} .
$$

Denote for a moment such modified sequence by $\left\{\bar{u}_{n}\right\}_{n \in \mathbb{N}_{0}}$ and the corresponding sequence (29) by $\left\{\bar{r}_{n}\right\}_{n \in \mathbb{N}_{0}}$. Since we changed only finitely many components of the sequence $\left\{u_{n}\right\}_{n \in \mathbb{N}_{0}},\left\{\bar{u}_{n}\right\}_{n \in \mathbb{N}_{0}} \in l^{1}$. Also, as only a finite number of elements of $\left\{r_{n}\right\}_{n \in \mathbb{N}_{0}}$ were changed, $\left\{\bar{r}_{n}\right\}_{n \in \mathbb{N}_{0}}$ converges (to the same limit) so that $\left\{\bar{u}_{n}\right\}_{n \in \mathbb{N}_{0}} \in D_{\max }$.

Since there are a finite number of positive $\bar{r}_{n}=-\lambda_{n} \bar{u}_{n}+\mu_{n+1} \bar{u}_{n+1}$, their minimum is positive and $\inf \left\{\bar{r}_{0}, \ldots, \bar{r}_{n_{0}-1}, r_{n_{0}} \ldots\right\}=r>0$. Thus, we can take a nonnegative sequence $\left\{u_{n}\right\}_{n \in \mathbb{N}_{0}} \in D_{\max }$ with the associated sequence $\left\{r_{n}\right\}_{n \in \mathbb{N}_{0}}$ satisfying $\inf \left\{r_{n}\right\}_{n \in \mathbb{N}_{0}}=r>0$. Since $\sum_{n=0}^{\infty} u_{n}<+\infty$, and $r_{n} \geq r>0$ for $n \geq 0$ and $\lambda_{0} u_{0} \geq 0$, we must have

$$
\infty>\sum_{n=1}^{\infty} \frac{g_{n-1}}{\mu_{n}}\left(r_{0}+\lambda_{0} u_{0}+\sum_{i=1}^{n} r_{i} g_{i}^{-1}\right) \geq r \sum_{n=1}^{\infty} \frac{g_{n-1}}{\mu_{n}}\left(\sum_{i=1}^{n} g_{i}^{-1}\right),
$$

so that the series in $(28)$ is convergent.

To prove the converse, define $u_{n}$ by (29) with arbitrary $\left\{r_{n}\right\}_{n \in \mathbb{N}_{0}}$ converging to $I>$ 0 (e.g. we may take $r_{n}=I$ for all $n$ ). By (28) such defined $\left\{u_{n}\right\}_{n \in \mathbb{N}_{0}} \in l^{1}$, so that $\left\{u_{n}\right\}_{n \in \mathbb{N}_{0}} \in D_{\max }$ and since $I>0$, the assertion follows by (23).

REMARK 2. The condition (28) is exactly the condition of [1, Chapter 3, Theorem 2.3] for the uniqueness of minimal dishonest solutions to the forward equation. In the present context this result is stronger as it gives uniqueness among all possible $l^{1}$ solutions.

We complete the paper by giving a survey of standard cases that are covered by Theorems 5 and 6 .

OBservation 1. If both sequences $\left\{\mu_{n}^{-1}\right\}_{n \in \mathbb{N}},\left\{\lambda_{n}^{-1}\right\}_{n \in \mathbb{N}} \notin l^{1}$, then $T=\overline{T_{\min }}=T_{\max }$. In particular, this is true for the standard birth-and-death problem from population theory where the coefficients are affine functions of $n$.

Proof. Expanding (28) we get for a given $n$

$$
\frac{1}{\mu_{n}}\left(1+\frac{\lambda_{n-1}}{\mu_{n-1}}+\ldots+\frac{\lambda_{n-1} \ldots \lambda_{1}}{\mu_{n-1} \ldots \mu_{1}}\right) \geq \frac{1}{\mu_{n}} .
$$

Similarly, expanding (18) we get

$$
\frac{1}{\lambda_{n}}\left(1+\frac{\mu_{n+1}}{\lambda_{n+1}} \ldots\right)
$$

and putting these together we get the divergence of both series. 
OBSERVATion 2. If $\left\{\mu_{n}^{-1}\right\}_{n \in \mathbb{N}} \in l^{1}$ and

$$
\lim _{n \rightarrow \infty} \frac{\lambda_{n}}{\mu_{n}}=q<1
$$

then $T=\overline{T_{\min }} \neq T_{\max }$.

Proof. From (32), $\lambda_{n} / \mu_{n} \leq q_{0}<1$ starting from some $n_{0}$. Thus

$$
\frac{1}{\mu_{n}}\left(1+\frac{\lambda_{n-1}}{\mu_{n-1}}+\ldots+\frac{\lambda_{n-1} \ldots \lambda_{1}}{\mu_{n-1} \ldots \mu_{1}}\right) \leq \frac{1}{\mu_{n}}\left(1+q_{0}+M_{n_{0}} q_{0}^{n}\right),
$$

where $M_{n_{0}}$ does not depend on $n$. Since $q_{0}<1$, the series (28) is convergent. Similarly, (18) is satisfied as it involves $\frac{\mu_{n}}{\lambda_{n}} \geq q_{0}>1$ which gives the divergence of the inner series.

OBSERVATION 3. If the sequence $\left\{\mu_{n}\right\}_{n \in \mathbb{N}}$ is of polynomial growth: $\mu_{n}=O\left(n^{\beta}\right)$ for some $\beta$ as $n \rightarrow \infty,\left\{\lambda_{n}^{-1}\right\}_{n \in \mathbb{N}} \in l^{1}$ and

$$
\lim _{n \rightarrow \infty} \frac{\lambda_{n}}{\mu_{n}}=q>1
$$

then $\overline{T_{\min }} \varsubsetneqq T=T_{\max }$.

Proof. As in the above proof

$$
\frac{1}{\mu_{n}}\left(1+\frac{\lambda_{n-1}}{\mu_{n-1}}+\ldots+\frac{\lambda_{n-1} \ldots \lambda_{1}}{\mu_{n-1} \ldots \mu_{1}}\right) \geq \frac{1}{\mu_{n}}\left(1+q_{0}+M_{n_{0}} q_{0}^{n}\right),
$$

for some $n_{0}$ and $q_{0}>1$, where $M_{n_{0}}$ does not depend on $n$. Since $q_{0}>1, q_{0}^{n} / n^{\beta}$ diverges for any $\beta$ and the series (28) diverges. Similarly, the series in (18) is summable.

OBservation 4. There are sequences $\left\{\lambda_{n}\right\}_{n \in \mathbb{N}_{0}}$ and $\left\{\mu_{n}\right\}_{n \in \mathbb{N}_{0}}$ for which $\overline{T_{\min }} \varsubsetneqq T \varsubsetneqq$ $T_{\max }$.

Proof. Take $\lambda_{n}=2 \cdot 3^{n}$ and $\mu_{n}=3^{n}$. Terms in the series (18) are

$$
\frac{1}{2 \cdot 3^{n}}\left(1+\frac{1}{2}+\frac{1}{2^{2}} \ldots\right)
$$

so that the series is summable. Terms in the series (28) are

$$
\frac{1}{3^{n}}\left(1+2+\ldots+2^{n-1}\right)
$$

so that this series is also summable.

\section{References}

[1] W. J. Anderson, Continuous-Time Markov Chains: an Applications-Oriented Approach, Springer Series in Statistics, Springer, New York, 1991.

[2] L. Arlotti, A perturbation theorem for positive contraction semigroups on $L^{1}$-spaces with applications to transport equations and Kolmogorov's differential equations, Acta Appl. Math. 23 (1991), 129-144.

[3] J. Banasiak, On an extension of Kato-Voigt perturbation theorem for substochastic semigroups and its applications, Taiwanese J. Math. 5 (2001), 169-191.

[4] J. Banasiak, On a non-uniqueness in fragmentation models, Math. Methods Appl. Sci. 25 (2002), 541-556. 
[5] J. Banasiak, On well-posedness of a Boltzmann like semiconductor model, Math. Models Methods Appl. Sci. 13 (2003), 875-892.

[6] A. T. Bharucha-Reid, Elements of the Theory of Markov Processes and Their Applications, McGraw-Hill, New York, 1960.

[7] S. N. Elaydi, An Introduction to Difference Equations, Springer, New York, 1999.

[8] G. Frosali, C. van der Mee and F. Mugelli, A characterization theorem for the evolution semigroup generated by the sum of two unbounded operators, Math. Methods Appl. Sci., to appear.

[9] E. Hille and R. S. Phillips, Functional Analysis and Semi-groups, Colloq. Publ. 31, Amer. Math. Soc., Providence, 1957.

[10] G. E. H. Reuter and W. Lederman, On differential equations for the transition probabilities of Markov processes with denumerably many states, Proc. Cambridge Phil. Soc. 49 (1953), $247-262$.

[11] G. E. H. Reuter, Denumerable Markov processes and the associated contraction semigroup, Acta Math. 97 (1957), 1-46.

[12] L. M. Ricciardi, Stochastic population theory: birth and death processes, in: Mathematical Ecology, T. G. Hallamand and S. A. Levin (eds.), Springer, Berlin, 1986, 155-190.

[13] J. Voigt, On substochastic $C_{0}$-semigroups and their generators, Transport Theory Statist. Phys. 16 (1987), 453-466. 